\title{
The anisotropy of local turbulence in the Earth's core
}

\author{
Masaki Matsushima*, Takahiro Nakajima**, and Paul H. Roberts \\ Institute of Geophysics and Planetary Physics, University of California, Los Angeles, CA 90095, U.S.A.
}

(Received July 29, 1998; Revised February 15, 1999; Accepted March 24, 1999)

\begin{abstract}
The anisotropy of local turbulence in the Earth's core is examined. It is recognized that small-scale motions in the core are strongly influenced by the Earth's rotation and its magnetic field. A small region of the core is simulated (the computational box), across which the prevailing large-scale (toroidal) magnetic field is supposed to be uniform and in which the temperature or compositional gradient providing the buoyancy that powers the turbulence is parallel to the (uniform) gravitational field. The simulations are used to estimate the turbulent fluxes of mean fields and their dependence on the latitude at which the computational box is situated. It is found that the effect of local turbulence on the diffusion of large-scale fields is significant, and that turbulent transport is anisotropic. It is believed that the results of the present study will prove useful in determining geophysically realistic diffusivities for use in future global geodynamo simulations.
\end{abstract}

\section{Introduction}

The molecular kinematic viscosity, and the thermal and compositional diffusivities of the Earth's core are very small. It is therefore very likely that large-scale differences in momentum density, entropy density and chemical composition are diffused much more effectively by small-scale turbulence than by molecular processes (Braginsky, 1964). To be realistic, simulations of the magnetohydrodynamics of the core and of the geodynamo must either resolve the small-scale motions numerically, an impossible task at present, or parameterize their effects on the macroscales in a plausible way. Every published geodynamo simulation has replaced the molecular diffusivities by much larger eddy diffusivities. Even this has not sufficed, and it has been necessary to supplement viscosity with hyperdiffusivity (e.g., Glatzmaier and Roberts, 1995a,b; Kuang and Bloxham, 1997).

The representation of unresolved scales by enhanced diffusivities is an idea that, through Osborne Reynolds' treatment of shear turbulence, goes back to the nineteenth century. It is the cornerstone of what is sometimes called 'local turbulence theory'. A local turbulence model is one in which, as for molecular transport, the fluxes sought are at each point dependent only on the gradients of the relevant macroscale fields at that point. The turbulent fluxes are then represented by eddy diffusivities. Lacking a developed theory of MHD turbulence in rapidly rotating fluids, it is natural to hope that a local turbulence model will suffice to describe core turbulence.

In the simulations cited above, the local turbulence approach is implicitly invoked. The turbulent fluxes take the

*Now at Department of Earth and Planetary Sciences, Tokyo Institute of Technology, Tokyo 152-8551, Japan.

** Now at Tono Geoscience Center, Japan Nuclear Cycle Development Institute, Toki, Gifu 509-5102, Japan.

Copy right $($ C The Society of Geomagnetism and Earth, Planetary and Space Sciences (SGEPSS); The Seismological Society of Japan; The Volcanological Society of Japan; The Geodetic Society of Japan; The Japanese Society for Planetary Sciences. same form as the relatively insignificant molecular fluxes, i.e., turbulent diffusion is supposed, like molecular diffusion, to be as isotropic, but with greatly enhanced diffusivities. The results of the simulations have been encouraging. Many of the characteristics of the main geomagnetic field have been approximately reproduced in magnitude and time scale, e.g., polarity reversals, westward drift, etc. One might say of the models that their success is unexpected, bearing in mind that they are firmly based on isotropic turbulent diffusion. In reality, because the small-scale motions are strongly influenced by the Earth's rotation and its magnetic field and are highly anisotropic, turbulent transport should be represented not by scalar diffusivities but by highly anisotropic tensor diffusivities. Hyperdiffusivity, as applied for example by Glatzmaier and Roberts, acts (through the spherical harmonic components) primarily on the horizontal scales. This is certainly some kind of anisotropy, but one introduced from computational expediency rather than physical necessity; the preferred directions of turbulent diffusion should relate to the directions of the rotation axis and the magnetic field (see below), not only to the direction of gravity. It should also be noted that Zhang and Jones (1997) argue cogently that hyperviscosity affects the dynamics of convection significantly wherever the magnetic field is weak.

Braginsky and Meytlis (1990) took the first steps in determining anisotropic diffusivity tensors. They found, from the linearized convection equations, that the most unstable motions have a small wavelength in the direction perpendicular to the rotation axis and to the direction of the prevailing magnetic field, i.e., the small scale convection cells are 'platelike'. St. Pierre (1996) provided corroboration. He examined the evolution of a spherical buoyant blob as it rises through the core. He found that the blob quickly broke up into platelike structures, elongated in the directions of the rotation axis and field. Braginsky and Meytlis also developed a heuristic theory of the turbulence that arises when the buoyancy gradient is increased well beyond critical. They made estimates of 
the turbulent diffusivities in the three principal directions of the diffusivity tensors, and found them to be very different. Their work was developed further by Braginsky and Roberts (1995).

In an attempt to improve on some of the heuristic features of the Braginsky-Meytlis theory, we attempt in this paper to simulate anisotropic buoyancy-driven turbulence in the core by direct numerical simulation (DNS). The length scale of the local turbulence considered here is so small, of the order of kilometer (see below), that it is impossible to resolve it by global simulations. By the same token, we may assume that the macroscale velocity, magnetic fields and temperature gradient merely provide a uniform background on which the turbulence rides, this turbulence being driven by thermal buoyancy rather than shear. We regard deviations from the uniform background, arising perhaps from macroscopic velocity or field gradients, or from geometrical effects associated with the curvature of the core, as small secondorder effects that can be ignored in a first approximation when describing core turbulence, even though these effects are very significant for the macroscale fields; e.g., see Zhang (1999). We shall represent a small region in the outer core by a rectangular box, moving with the mean flow, and having faces in the coordinate planes of a locally Cartesian frame $(x, y, z)$. We first perform simple linear analyses for the marginal state and (following Braginsky and Meytlis, 1990) for the state that has the maximum growth rate. We then investigate numerically the turbulent magnetoconvection that arises in supercritical conditions, and use the resulting solutions to estimate quantitatively the effect of the motions on the turbulent transport of mean fields.

Our quantitative assessment of the turbulent transport coefficients could be used in global simulations of the geodynamo. We believe that these would be more realistic than those that assumed isotropic turbulent diffusion. Such simulations have not yet been attempted.

\section{Linear Analyses of Instability}

The basic equations to be solved are

$$
\begin{aligned}
& \partial_{t} \mathbf{V}+(\mathbf{V} \cdot \nabla) \mathbf{V}=-\rho^{-1} \nabla P-2 \mathbf{\Omega} \times \mathbf{V} \\
&+\left(\mu_{0} \rho\right)^{-1}(\nabla \times \mathbf{B}) \times \mathbf{B} \\
&+C \mathbf{g}+v \nabla^{2} \mathbf{V}, \\
& \partial_{t} \mathbf{B}=\nabla \times(\mathbf{V} \times \mathbf{B})+\eta \nabla^{2} \mathbf{B}, \\
& \partial_{t} C+(\mathbf{V} \cdot \nabla) C=\kappa \nabla^{2} C, \\
& \nabla \cdot \mathbf{V}=0, \quad \nabla \cdot \mathbf{B}=0,
\end{aligned}
$$

where $\mathbf{V}$ is the velocity field in a coordinate system rotating with the angular velocity $\boldsymbol{\Omega}, \mathbf{B}$ the magnetic field, $C$ the buoyancy source energizing the convection (called the 'codensity' by Braginsky and Roberts, 1995), $P$ the pressure, $\rho$ the density of the fluid (assumed to be incompressible in the Boussinesq sense), $\mu_{0}$ the magnetic permeability of free space, $\mathbf{g}$ the gravitational acceleration vector, $v$ the kinematic viscosity, $\eta$ the magnetic diffusivity, and $\kappa$ the diffusivity for $C$. In this paper, we simply consider a thermally convective system. Then the co-density $C$ can be expressed in terms of the deviation, $\Theta$, of the temperature from a reference field, and the coefficient of thermal expansion, $\alpha_{T}$, by $C=-\alpha_{T} \Theta$.
The theory can be easily generalized to the case in which buoyancy is also supplied, partially or completely, by differences in chemical composition (Braginsky and Roberts, 1995).

As mentioned in the last section, we represent a small region in the outer core by a rectangular box and impose periodic boundary conditions. We adopt a locally Cartesian frame, $(x, y, z)$, that moves with the local macroscale velocity, i.e., in this frame the effects of global motion are, to a first approximation, removed. The directions of the coordinate axes are chosen so that $\boldsymbol{\Omega}=\mathbf{1}_{z} \Omega$, and $\mathbf{1}_{y}$ is in the direction of increasing longitude. (We use $\mathbf{1}_{k}$ to denote the unit vector in the $k$-direction.) We impose a uniform magnetic field, $\mathbf{B}_{0}$, to represent a strong toroidal magnetic field which is supposed to be generated by the global dynamo. We take this to be $\mathbf{B}_{0}=\mathbf{1}_{y} B_{0}$, where $B_{0}$ is a constant. Figure 1 presents schematic pictures of the computational box used in our study.

Now we separate the velocity field, the magnetic field, the buoyancy field, and the pressure into mean and fluctuating parts as

$$
\begin{aligned}
& \mathbf{V}=\overline{\mathbf{V}}+\mathbf{v}^{\prime}, \quad \mathbf{B}=\overline{\mathbf{B}}+\mathbf{b}^{\prime}, \\
& \Theta=\bar{\Theta}+\theta^{\prime}, P=\bar{P}+p^{\prime},
\end{aligned}
$$

where the overbar denotes mean fields and the prime fluctuating fields. In this study, the ensemble mean for a field is its average over the computational box; for example, $\overline{\mathbf{v}^{\prime}}=\mathbf{0}$.

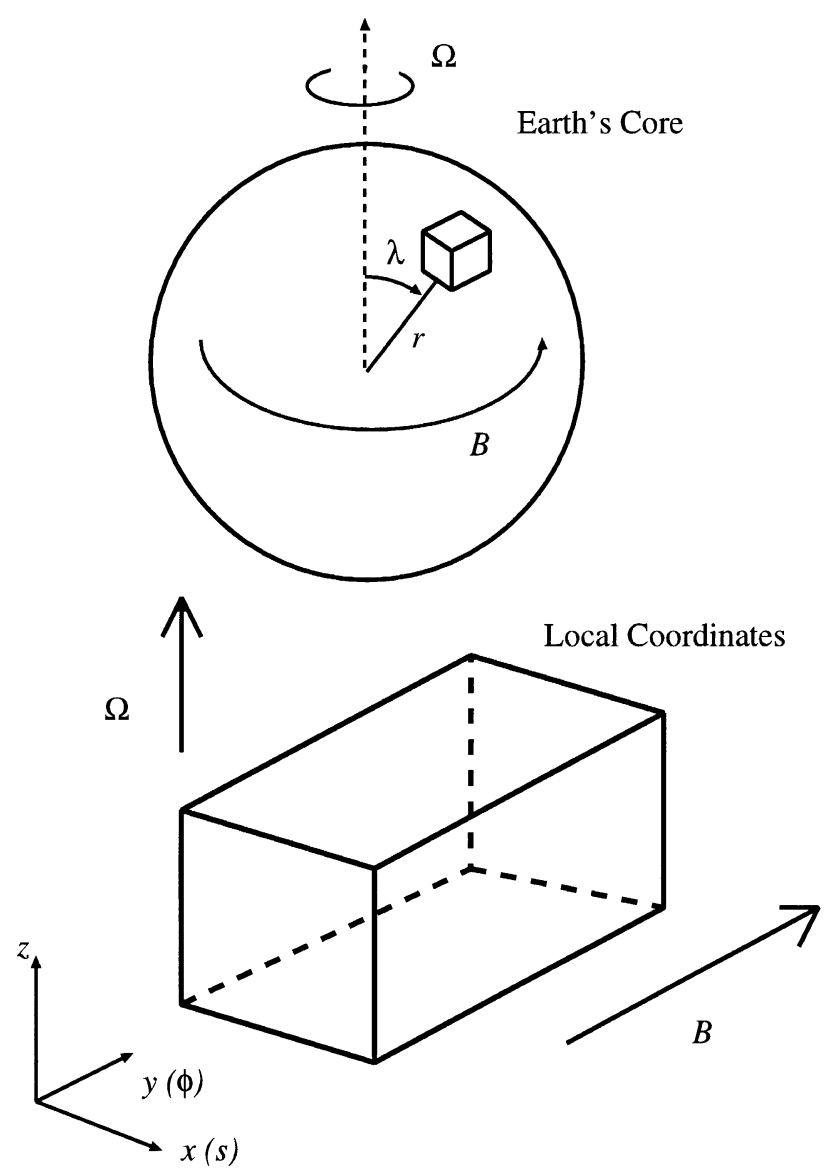

Fig. 1. Schematic pictures of the computational box and its relationship to the Earth's core. 
We also have (see above) $\overline{\mathbf{V}}=\mathbf{0}$.

Scaling length by $a$, which is a typical length of the computational box, time by $a^{2} / \eta$, velocity by $\eta / a$, magnetic field by $B_{0}$, temperature by $\beta a$, where $\beta$ is the large-scale buoyancy gradient, and pressure by $2 \Omega \eta \rho$, we find that the fluctuating parts of (1)-(3) are

$$
\begin{aligned}
& \varepsilon_{\eta}\left\{\partial_{t} \mathbf{v}+(\mathbf{v} \cdot \nabla) \mathbf{v}\right\}=-\nabla p-\mathbf{1}_{z} \times \mathbf{v} \\
&+\Lambda(\nabla \times \mathbf{b}) \times\left(\mathbf{1}_{y}+\mathbf{b}\right) \\
&+\operatorname{Ra} \theta \mathbf{1}_{r}+\varepsilon_{v} \nabla^{2} \mathbf{v}, \\
& \partial_{t} \mathbf{b}+(\mathbf{v} \cdot \nabla) \mathbf{b}=\left\{\left(\mathbf{1}_{y}+\mathbf{b}\right) \cdot \nabla\right\} \mathbf{v}+\nabla^{2} \mathbf{b}, \\
& \partial_{t} \theta+\mathbf{v} \cdot \nabla \theta=-\mathbf{v} \cdot \nabla \bar{\Theta}+P q \nabla^{2} \theta, \\
& \nabla \cdot \mathbf{v}=0, \quad \nabla \cdot \mathbf{b}=0,
\end{aligned}
$$

where $\mathbf{g}=-g \mathbf{1}_{r}$ and $(r, \vartheta, \phi)$ are spherical coordinates. The primes are omitted from (6)-(9) for simplicity. The nondimensional numbers are the Rayleigh number, $R a$, the Elsasser number, $\Lambda$, the Ekman number, $\varepsilon_{v}$, the magnetic Ekman number, $\varepsilon_{\eta}$, and the diffusivity ratio, $P q$, defined by

$$
\begin{aligned}
& R a=\frac{g \alpha_{T} \beta a^{2}}{2 \Omega \eta}, \Lambda=\frac{B_{0}^{2}}{2 \Omega \eta \mu_{0} \rho}, \\
& \varepsilon_{v}=\frac{v}{2 \Omega a^{2}}, \varepsilon_{\eta}=\frac{\eta}{2 \Omega a^{2}}, P q=\frac{\kappa}{\eta} .
\end{aligned}
$$

The large-scale temperature field, $\bar{\Theta}$, drives the flow.

We first perform a linear analysis by neglecting the nonlinear terms in (6)-(8). Only $\partial_{t}$ in (8) is retained as in Braginsky and Meytlis (1990). They estimated a growth rate $\gamma$ (see below) and compared it with typical diffusion rates for the velocity and the magnetic fields. It was assumed that the magnetic diffusivity is larger than those for the velocity field and the co-density. The magnetic diffusion rate is therefore much larger than $\gamma$, and $\partial_{t}$ in (7) was neglected. Although the kinematic viscosity can be as small as the co-density diffusion, the magnetic friction which acts on the velocity field is so large that $\partial_{t}$ in (6) was also neglected. The resulting equations are

$$
\begin{gathered}
\mathbf{0}=-\nabla p-\mathbf{1}_{z} \times \mathbf{v}+\Lambda(\nabla \times \mathbf{b}) \times \mathbf{1}_{y}+R a \theta \mathbf{1}_{r}+\varepsilon_{v} \nabla^{2} \mathbf{v}, \\
\mathbf{0}=\mathbf{1}_{y} \cdot \nabla \mathbf{v}+\nabla^{2} \mathbf{b} \\
\partial_{t} \theta=-\mathbf{v} \cdot \nabla \bar{\Theta}+P q \nabla^{2} \theta
\end{gathered}
$$

Assuming that $\mathbf{v}, \mathbf{b}$ and $\theta$ are proportional to $\exp (i \mathbf{k} \cdot \mathbf{r}+$ $\gamma t)=\exp \left\{i\left(k_{x} x+k_{y} y+k_{z} z\right)+\gamma t\right\}$, we have $\mathbf{b}=\left(i k_{y} / k^{2}\right) \mathbf{v}$ from (12) and $\theta=\left(v_{x} \sin \lambda+v_{z} \cos \lambda\right) /\left(\gamma+P q k^{2}\right)$ from (13), where it is assumed that $\nabla \bar{\Theta}$ is parallel to $\mathbf{g}(\nabla \bar{\Theta}=$ $-\mathbf{1}_{r}=-\mathbf{1}_{x} \sin \lambda-\mathbf{1}_{z} \cos \lambda$ in a nondimensional form), $\lambda$ is the angle between $\mathbf{1}_{z}$ and $\mathbf{1}_{r}$ (i.e., the colatitude of the region in the core where the computation box is supposed situated), and $k^{2}=k_{x}^{2}+k_{y}^{2}+k_{z}^{2}$. We obtain

$$
\gamma=\frac{\operatorname{Ra\gamma }_{*}\left\{\left(k_{x} \cos \lambda-k_{z} \sin \lambda\right)^{2}+k_{y}^{2}\right\}}{k^{2} \gamma_{*}^{2}+k_{z}^{2}}-P q k^{2},
$$

where

$$
\gamma_{*}=\Lambda \frac{k_{y}^{2}}{k^{2}}+\varepsilon_{\nu} k^{2}
$$

The critical Rayleigh number, $R a_{\mathrm{cr}}$, is derived from (14), by setting $\gamma$ at zero for a marginal state,

$$
R a_{\mathrm{cr}}=P q \frac{k^{2} \gamma_{*}^{2}+k_{z}^{2}}{\gamma_{*}} \frac{k^{2}}{\left(k_{x} \cos \lambda-k_{z} \sin \lambda\right)^{2}+k_{y}^{2}} .
$$

Braginsky and Meytlis (1990) considered that the Coriolis and the Lorentz forces do not stabilize the fluid motion but decrease the growth rate, $\gamma$. They presumed that the elongation of a convective cell in the $z$-direction reduces the influence of the Coriolis force and its elongation in the direction of the strong magnetic field, the $y$-direction here, reduces the magnetic friction. They also argued that these conclusions are insensitive to latitude when $\nabla \bar{\Theta}$ is parallel to g. Hence they pictured the core as filled with turbulent plate-like cells.

Here we quantitatively derive, in terms of the appropriate nondimensional numbers, the shapes of convective cells, both for the marginal states and for the modes that have the maximum growth rate. The shape is defined by the wavelengths in the $x, y$ and $z$ directions given by $\ell_{x}=2 \pi /\left|k_{x}\right|$, $\ell_{y}=2 \pi /\left|k_{y}\right|$ and $\ell_{z}=2 \pi /\left|k_{z}\right|$. It is difficult to believe that very long, slender convection cells can form and survive for a long time in the core. If, however, we seek the values of $k_{x}$, $k_{y}$ and $k_{z}$ that minimize $R a_{\mathrm{cr}}$ for given $\Lambda, \varepsilon_{\nu}$ and $P q$, by solving $\partial R a_{\mathrm{cr}} / \partial k_{x}=\partial R a_{\mathrm{cr}} / \partial k_{y}=\partial R a_{\mathrm{cr}} / \partial k_{z}=0$, we discover that $k_{z}=0$ when $\lambda=0\left(k_{x} \neq 0\right.$ and $\left.k_{y} \neq 0\right)$. This difficulty was raised by Braginsky (1964), and is also mentioned by Braginsky and Roberts (1995), who ask 'How long are the cells in the $z$-direction?', and 'What is the mechanism that limits their length $\ell_{z}$ ?' To make progress, we must postulate a unit of length for the convective cells, so we here take $\ell_{z}$ as that length, even though the shape and size of the cells may depend on the location of the computational box in the core, as defined by $\lambda$.

Figure 2 shows, for $\Lambda=10, \varepsilon_{v}=10^{-3}$ and $P q=10^{-1}$, the latitudinal dependence of the shape of the convective cells in the marginal state. It is clearly seen that $\ell_{y}$ does not change very much, whereas $\ell_{x}$ becomes larger as the computational box approaches the equator, where $\ell_{z}$ is fixed at unity. In particular, $k_{x} \rightarrow 0$, or $\ell_{x} \rightarrow \infty$ as $\lambda \rightarrow \pi / 2$. (This case is not shown in the figure.) It is found that $\ell_{y}$ ranges from between 2 to 4 times larger than $\ell_{z}$. This is caused by the magnetic field imposed in the $y$-direction. In contrast, $\ell_{x}$ is a little smaller than $\ell_{z}$ for $\lambda \leq \pi / 12$, but becomes large with increasing $\lambda$, as mentioned above. In the marginal state, therefore, the convective cells are not platelike.

The shape of the convective cells that maximize the growth rate differs from that of the marginal state. We show, in Fig. 3, the latitudinal dependence of their shape for $\left(R a, \varepsilon_{v}\right)=$ $\left(40,10^{-3}\right),\left(40,10^{-5}\right)$, and $\left(160,10^{-5}\right)$, with $\Lambda=10$ and $P q=10^{-1}$. The difference between $\ell_{y}$ for $\varepsilon_{v}=10^{-3}$ and for $\varepsilon_{v}=10^{-5}$ is clear. Generally the cells that maximize the growth rate are smaller than those of the marginal state. It is found that $\ell_{y}$ is always larger than $\ell_{z}=1$, whereas from the pole $(\lambda=0)$ to mid-latitudes $\ell_{x}$ is smaller. As for the marginal state, $\ell_{x}$ becomes larger with increasing $\lambda$, but this tendency is less marked than for the marginal state.

For $\lambda=0$ and $\varepsilon_{v}=10^{-5}$, the marginal Rayleigh number is $R a_{\text {cr }}=20.5136$, the corresponding cell being $\left(\ell_{x}, \ell_{y}, \ell_{z}\right)=$ $(0.7398,2.405,1)$. When $R a=40 \approx 2 R a_{\mathrm{cr}}$, the maximum growth rate, $\gamma=17.92$, arises for the cell $\left(\ell_{x}, \ell_{y}, \ell_{z}\right)=$ 


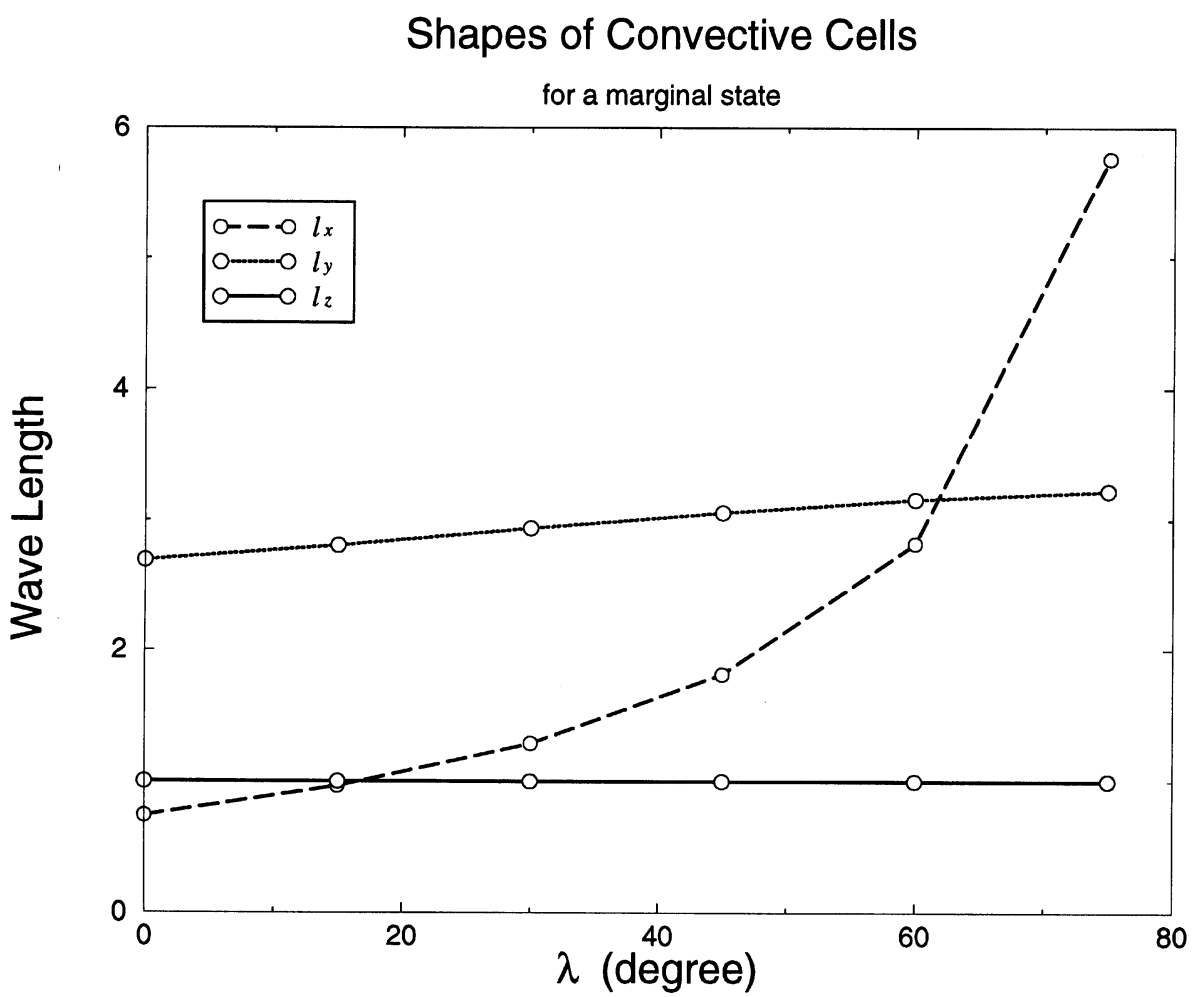

Fig. 2. The latitudinal dependence of the shape of convective cells in the marginal state for $\Lambda=10^{+1}, \varepsilon_{v}=10^{-3}$, and $P q=10^{-1}$.

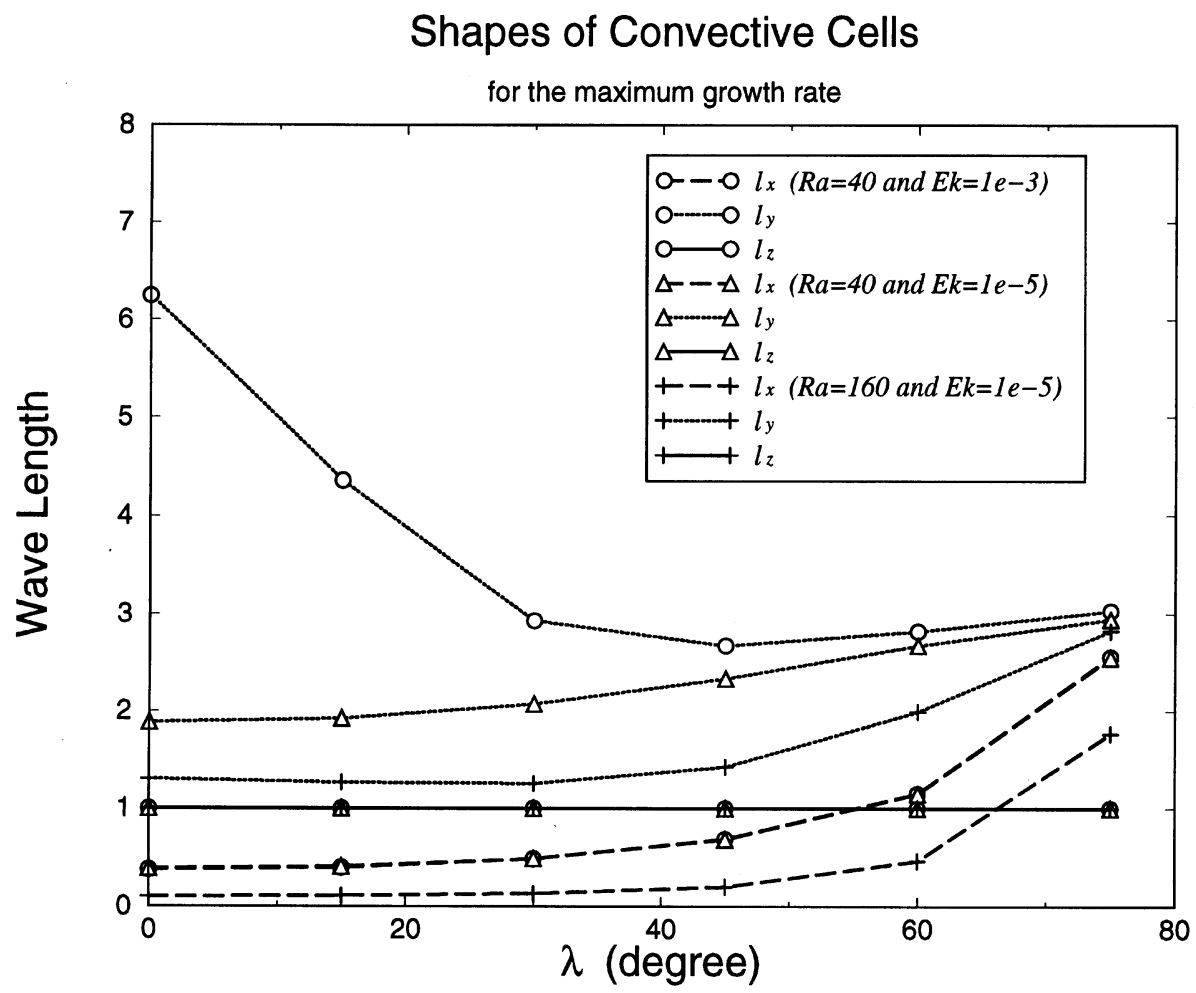

Fig. 3. The latitudinal dependence of the shape of convective cells for which the growth rate is a maximum for $\left(R a, \varepsilon_{v}\right)=\left(40,10^{-3}\right),\left(40,10^{-5}\right)$, and $\left(160,10^{-5}\right)$ with $\Lambda=10^{+1}$ and $P q=10^{-1}$. 
$(0.3830,1.883,1)$, which does not so much resemble a plate as it does a long matchbox. When $R a=160 \approx 8 R a_{\mathrm{cr}}$, however, the cells grow fastest in polar regions; the maximum growth rate, $\gamma=397.4$, occurs for the cells $\left(\ell_{x}, \ell_{y}, \ell_{z}\right)=$ $(0.09851,1.301,1)$ which are indeed platelike, having comparable dimensions in the $y$ - and $z$-directions, but which are about ten times smaller in the $x$-direction. This case is consistent with the conclusions of Braginsky and Meytlis (1990): platelike cells sometimes arise preferentially in the highly turbulent state.

When the convective box is situated at mid-latitude, for example at $\lambda=\pi / 3$, the maximum growth rate for $\varepsilon_{v}=10^{-5}$ and $R a=80 \approx 8 R a_{\text {cr }}$ is $\gamma=47.1051$, the corresponding cell dimensions being $\left(\ell_{x}, \ell_{y}, \ell_{z}\right)=(0.7779,2.406,1)$. Even when $R a=160 \approx 16 R a_{\mathrm{cr}},\left(\ell_{x}, \ell_{y}, \ell_{z}\right)=$ $(0.4608,1.995,1)$. It therefore appears that platelike convective cells are not preferred at mid-latitude even for highly turbulent states. This conclusion differs from that of Braginsky and Meytlis (1990). In examining the anisotropy of local turbulence in the Earth's core, we are therefore motivated to take special account of the location of the computational box in the core (something that has not previously been done).

\section{Numerical Methods}

A control volume method and a pseudo-spectral method were both used to solve (6)-(9). In the control volume method, we represent the variables at staggered grid points (e.g., Arakawa, 1994); that is, the pressure and co-density are defined at $\left(x_{i}, y_{j}, z_{k}\right)$, where $x_{i}=\ell_{x} i / I^{*}\left(i=0, \ldots, I^{*}\right)$, $y_{j}=\ell_{y} j / J^{*}\left(j=0, \ldots, J^{*}\right)$, and $z_{k}=\ell_{z} k / K^{*}(k=$ $\left.0, \ldots, K^{*}\right)$, while $x$-, $y$ - and $z$-components of the velocity and the magnetic fields are defined at $\left(x_{i+1 / 2}, y_{j}, z_{k}\right)$, at $\left(x_{i}, y_{j+1 / 2}, z_{k}\right)$, and at $\left(x_{i}, y_{j}, z_{k+1 / 2}\right)$, respectively. The time step is set so as to satisfy the Courant condition. The SMAC (Simplified Marker and Cell) method is used to derive the pressure from the equation of motion (6) and the equation of continuity (9). In this computation, we used a multigrid method to solve the Poisson equation to correct the pressure which is then used to correct the velocity field. The magnetic field is also corrected so as to satisfy $\nabla \cdot \mathbf{b}=0$ in a similar way. It may be noted that the multigrid method is more efficient than successive over-relaxation (SOR).

The pseudo-spectral method we used is nearly identical to that of St. Pierre (1996). The velocity and the magnetic fields are decomposed into toroidal and poloidal parts, and their defining scalar fields and the temperature $\theta$ are expressed in terms of three-dimensional Fourier series, for example,

$$
\begin{aligned}
\theta(x, y, z, t)= & \sum_{l=-l^{*}}^{l^{*}} \sum_{m=-m^{*}}^{m^{*}} \sum_{n=-n^{*}}^{n^{*}} \hat{\theta}_{l m n}(t) \\
& \cdot \exp \left[2 \pi i\left(\frac{l x}{\ell_{x}}+\frac{m y}{\ell_{y}}+\frac{n z}{\ell_{z}}\right)\right] .
\end{aligned}
$$

Like St. Pierre (1996), we removed (see above) all $z$-independent components of the velocity $\mathbf{v}$; these resemble geostrophic modes. We did this by subtracting $\ell_{z}^{-1} \int_{0}^{\ell_{z}} \mathbf{v} d z$ from $\mathbf{v}$. Here $\ell_{z}$ is the unit of length that defines the size of the computational box.
We first tested the reliability of our computational methods. We used each method to derive a solution starting from the same initial state for $\lambda=0$ and $\left(\ell_{x}, \ell_{y}, \ell_{z}\right)=(1,1,1)$. The nondimensional parameters selected were $\varepsilon_{v}=10^{-3}$ $\varepsilon_{\eta}=10^{-2}, \Lambda=10^{+1}, P q=10^{-1}$, and $R a=120.8 \approx$ $4.25 R a_{\mathrm{cr}}$, corresponding to $\Omega=7.29 \times 10^{-5} \mathrm{rad} / \mathrm{s}, \eta=$ $10 v=10 \kappa=1.46 \mathrm{~m}^{2} / \mathrm{s}, a=10^{3} \mathrm{~m}, \mu_{0}=4 \pi \times 10^{-7} \mathrm{H} / \mathrm{m}$, $\rho=1.1 \times 10^{4} \mathrm{~kg} / \mathrm{m}^{3}$, and $B_{0}=5.42 \mathrm{mT}$. The numbers of grid points, $\left(I^{*}, J^{*}, K^{*}\right)$, were taken to be $(32,32,32)$ in the both computations, and for the pseudo-spectral method the maximum wave numbers $l^{*}, m^{*}$ and $n^{*}$, in the $x$-, $y$ - and $z$-directions were all taken to be 10 .

Figure 4 shows, for each of the two methods, the time evolutions of the components $\overline{\theta v_{x}}, \overline{\theta v_{y}}$ and $\overline{\theta v_{z}}$ of the turbulent flux

$$
\mathbf{I}=\overline{\theta \mathbf{v}}
$$

which is our main objective in this paper. Even though the variables are defined at different grid points in the two methods and even though the system is chaotic, the fluxes are very similar up to $t=0.05$, approximately. Subsequently the evolutions took slightly different courses, and we could
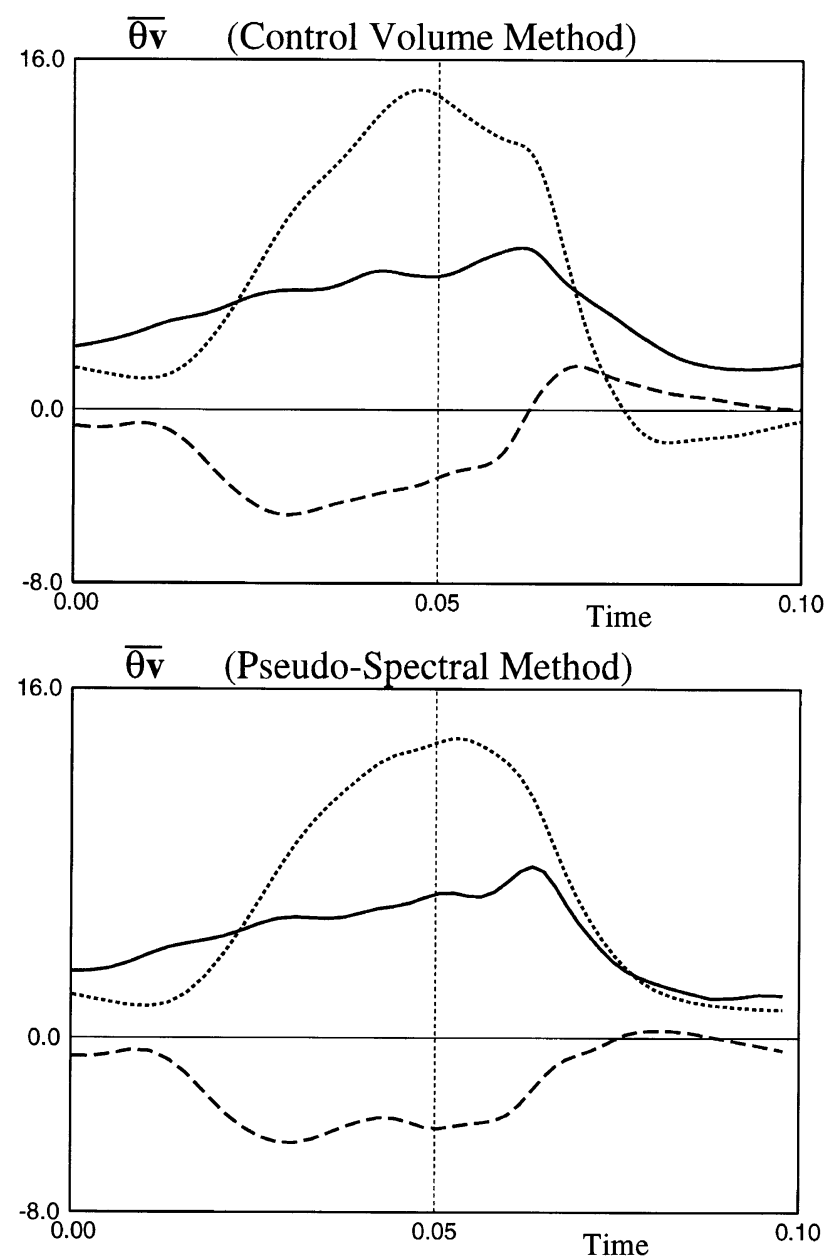

Fig. 4. Time evolutions of the components $\overline{\theta v_{x}}$ (broken lines), $\overline{\theta v_{y}}$ (dotted lines), and $\overline{\theta v_{z}}$ (solid lines) of the turbulent flux, according to both our control volume method and our spectral transform method. Parameters used are $\lambda=0,\left(\ell_{x}, \ell_{y}, \ell_{z}\right)=(1,1,1), \varepsilon_{v}=10^{-3}, \varepsilon_{\eta}=10^{-2}$ $\Lambda=10^{+1}, P q=10^{-1}$, and $R a=120 \approx 4.25 R a_{\mathrm{cr}}$. 
Table 1. Comparison between results from the control volume method and the pseudo-spectral method.

\begin{tabular}{cccc}
\hline & Control volume method & Pseudo-spectral method & $\begin{array}{c}\text { Control volume method } \\
\text { (another initial condition) }\end{array}$ \\
\hline$\left\langle\overline{\theta v_{x}}\right\rangle$ & $(-0.149 \pm 2.574) \times 10^{+0}$ & $(-0.180 \pm 1.994) \times 10^{+0}$ & $(1.326 \pm 3.809) \times 10^{+0}$ \\
$\left\langle\overline{\theta v_{y}}\right\rangle$ & $(0.300 \pm 6.040) \times 10^{+0}$ & $(0.579 \pm 5.041) \times 10^{+0}$ & $(-3.026 \pm 9.393) \times 10^{+0}$ \\
$\left\langle\overline{\theta v_{z}}\right\rangle$ & $(4.154 \pm 2.323) \times 10^{+0}$ & $(3.270 \pm 1.784) \times 10^{+0}$ & $(4.544 \pm 4.256) \times 10^{+0}$ \\
$\left\langle\overline{v_{x}^{2}}\right\rangle / 2$ & $(1.546 \pm 0.526) \times 10^{+2}$ & $(1.530 \pm 0.786) \times 10^{+2}$ & $(1.917 \pm 0.919) \times 10^{+2}$ \\
$\left\langle\overline{v_{y}^{2}}\right\rangle / 2$ & $(3.294 \pm 2.285) \times 10^{+2}$ & $(3.361 \pm 3.325) \times 10^{+2}$ & $(3.942 \pm 4.013) \times 10^{+2}$ \\
$\left\langle\overline{v_{z}^{2}}\right\rangle / 2$ & $(1.687 \pm 0.825) \times 10^{+2}$ & $(1.463 \pm 1.486) \times 10^{+2}$ & $(1.905 \pm 1.327) \times 10^{+2}$ \\
$\left\langle\overline{b_{x}^{2}}\right\rangle / 2$ & $(1.363 \pm 1.142) \times 10^{+1}$ & $(1.100 \pm 1.632) \times 10^{+1}$ & $(1.329 \pm 1.436) \times 10^{+1}$ \\
$\left\langle\overline{b_{y}^{2}}\right\rangle / 2$ & $(2.107 \pm 2.757) \times 10^{+1}$ & $(1.580 \pm 3.281) \times 10^{+1}$ & $(2.204 \pm 4.420) \times 10^{+1}$ \\
$\left\langle\overline{b_{z}^{2}}\right\rangle / 2$ & $(3.298 \pm 3.437) \times 10^{+1}$ & $(2.483 \pm 4.644) \times 10^{+1}$ & $(2.755 \pm 3.419) \times 10^{+1}$ \\
\hline
\end{tabular}

Table 2. Cartesian components of the turbulent flux.

\begin{tabular}{ccccc}
\hline$\lambda$ & $R a$ & $\left\langle I_{x}\right\rangle$ & $\left\langle I_{y}\right\rangle$ & $\left\langle I_{z}\right\rangle$ \\
\hline 0 & 40 & $(-0.718 \pm 8.824) \times 10^{-1}$ & $(+0.511 \pm 1.822) \times 10^{+0}$ & $(+2.003 \pm 0.593) \times 10^{+0}$ \\
$\pi / 6$ & 40 & $(+1.709 \pm 0.864) \times 10^{+0}$ & $(-4.828 \pm 2.569) \times 10^{+0}$ & $(+2.378 \pm 0.822) \times 10^{+0}$ \\
$\pi / 3$ & 40 & $(+3.888 \pm 1.311) \times 10^{+0}$ & $(-7.924 \pm 2.234) \times 10^{+0}$ & $(+2.384 \pm 0.631) \times 10^{+0}$ \\
0 & 102 & $(-0.277 \pm 3.877) \times 10^{+0}$ & $(+1.016 \pm 6.940) \times 10^{+0}$ & $(+4.836 \pm 4.149) \times 10^{+0}$ \\
$\pi / 3$ & 120 & $(+2.803 \pm 1.457) \times 10^{+1}$ & $(-3.966 \pm 2.368) \times 10^{+1}$ & $(+1.030 \pm 0.608) \times 10^{+1}$ \\
\hline
\end{tabular}

only compare the fluxes by statistical methods. We evaluated the ensemble averages of the turbulent fluxes $\left\langle\overline{\theta v_{x}}\right\rangle$, $\left\langle\overline{\theta v_{y}}\right\rangle$, and $\left\langle\overline{\theta v_{z}}\right\rangle$, where \langle\rangle denotes the ensemble average as averages over one magnetic diffusion time. The standard deviations of these quantities were also estimated. Table 1 gives these values and also the mean kinetic and magnetic energies $\left\langle\overline{v_{x}^{2}}\right\rangle / 2,\left\langle\overline{v_{y}^{2}}\right\rangle / 2,\left\langle\overline{v_{z}^{2}}\right\rangle / 2,\left\langle\overline{b_{x}^{2}}\right\rangle / 2,\left\langle\overline{b_{y}^{2}}\right\rangle / 2$, and $\left\langle\overline{b_{z}^{2}}\right\rangle / 2$. It is clear that the results from the two methods are in good agreement with one another. We therefore consider that our computation is reliable.

It should be noted that in order to compare magnetic energy $\mathbf{b}^{2} / 2$ with kinetic energy $\mathbf{v}^{2} / 2$ on the same scale, the magnetic energy has to be multiplied by $\Lambda / \varepsilon_{\eta}$; this has been done in preparing Table 1.

\section{Results and Discussion}

A strong toroidal magnetic field is supposed to be generated by the differential rotation in global dynamo processes, as mentioned in Section 2, and therefore $\Lambda=10$ is used in this paper. We also fixed the nondimensional parameters as $P q=10^{-1}, \varepsilon_{v}=10^{-3}$, and $\varepsilon_{\eta}=10^{-2}$. The Rayleigh number, $R a$, was set at $2 R a_{\mathrm{cr}} \sim 15 R a_{\mathrm{cr}}$. We then examined the time evolution of the magneto-convective turbulence for several choices of $\left(\ell_{x}, \ell_{y}, \ell_{z}\right)$ and colatitude $\lambda$. The integrations were continued for more than one magnetic diffusion time after the initial transients had disappeared.

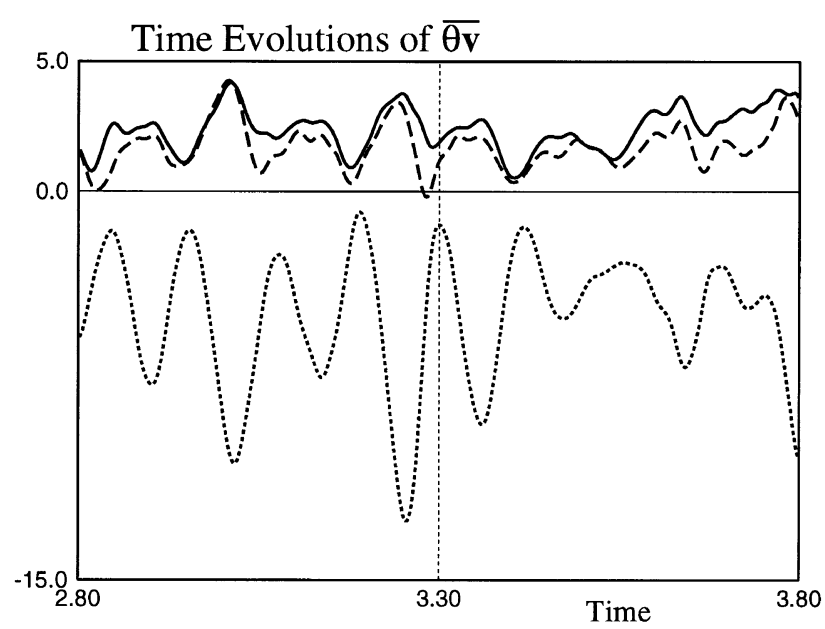

Fig. 5. Time evolutions of components of the turbulent flux, $\overline{\theta v_{x}}$ (broken line), $\overline{\theta v_{y}}$ (dotted line), and $\overline{\theta v_{z}}$ (solid line) for $\lambda=\pi / 6$ and $R a=40$.

As demonstrated above, since local turbulence in the core is expected to be anisotropic, the size of computational box is of importance. As an example, consider the case $\lambda=\pi / 6$. We first took $\left(\ell_{x}, \ell_{y}, \ell_{z}\right)=(0.4812,2.925,1)$ which corresponds to the cell size of maximum growth rate for $R a=40$. 
Table 3. The turbulent transport in the geophysical context.

\begin{tabular}{ccccc}
\hline$\lambda$ & $R a$ & $\langle I\rangle$ & $\langle\Psi\rangle$ & $\langle\Phi\rangle$ \\
\hline 0 & 40 & $(+2.755 \pm 1.065) \times 10^{+0}$ & $37.8^{\circ} \pm 15.6^{\circ}$ & $21.3^{\circ} \pm 97.2^{\circ}$ \\
$\pi / 6$ & 40 & $(+5.750 \pm 2.618) \times 10^{+0}$ & $63.1^{\circ} \pm 8.9^{\circ}$ & $-68.7^{\circ} \pm 9.5^{\circ}$ \\
$\pi / 3$ & 40 & $(+9.185 \pm 2.516) \times 10^{+0}$ & $74.7^{\circ} \pm 2.9^{\circ}$ & $-63.8^{\circ} \pm 4.9^{\circ}$ \\
0 & 102 & $(+7.079 \pm 7.399) \times 10^{+0}$ & $36.9^{\circ} \pm 14.0^{\circ}$ & $39.8^{\circ} \pm 92.4^{\circ}$ \\
$\pi / 3$ & 120 & $(+5.008 \pm 2.768) \times 10^{+1}$ & $78.0^{\circ} \pm 3.0^{\circ}$ & $-53.0^{\circ} \pm 8.2^{\circ}$ \\
\hline
\end{tabular}

\section{Trajectories of $\overline{\theta \mathbf{v}}$}
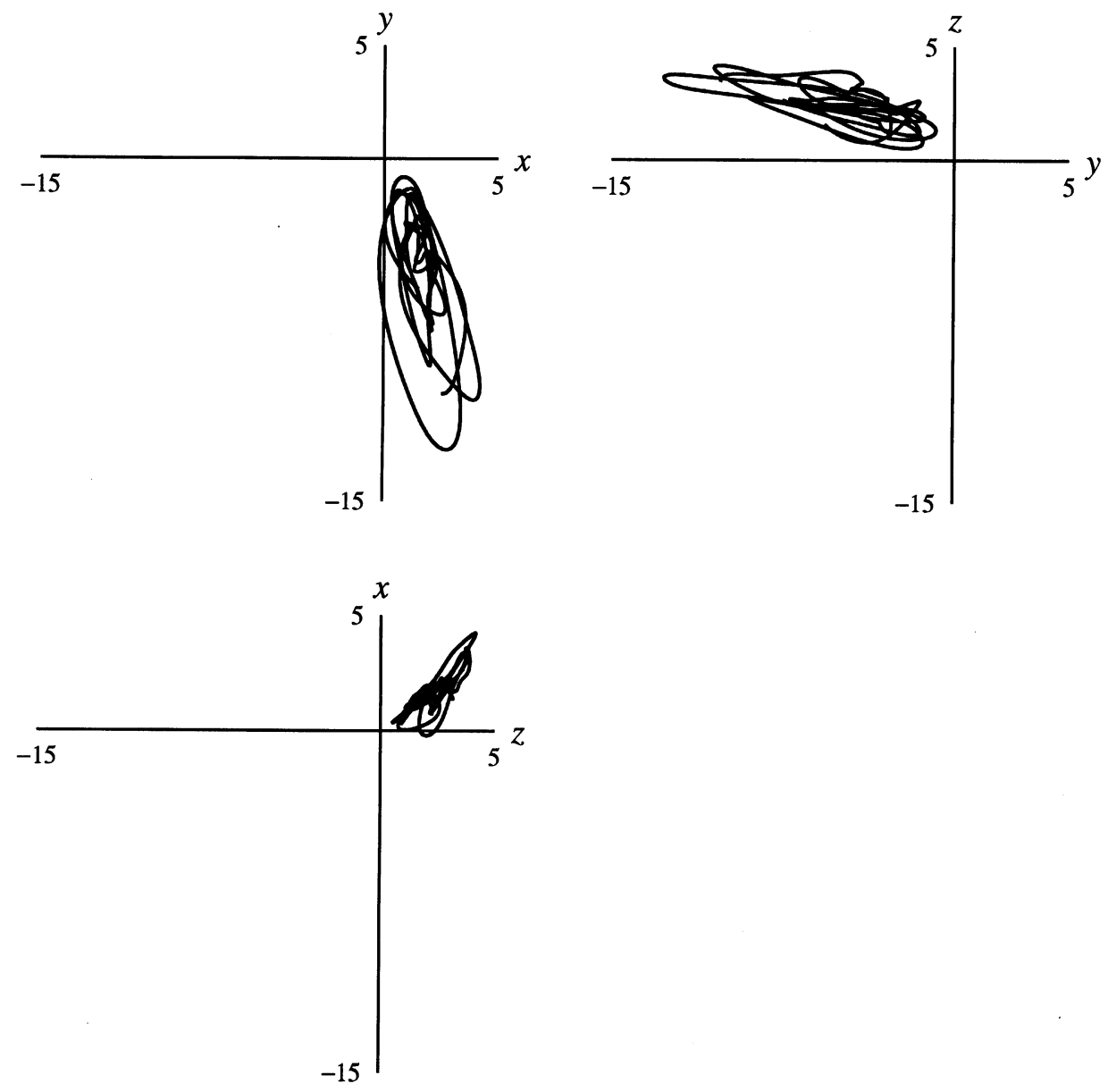

Fig. 6. Trajectories of the turbulent flux, $I_{x}, I_{y}$, and $I_{z}$ projected onto the $x y$-, $y z$ - and $z x$-planes for $\lambda=\pi / 6$ and $R a=40$.

We spectrally analyzed the computed velocity field and found that the kinetic energies in the $x$ - and $y$-directions were greatest at zero wave numbers. This indicates that the computational box is too small to simulate local turbulence. We next took $\left(\ell_{x}, \ell_{y}, \ell_{z}\right)=(0.9624,2.925,1)$; that is, a cell of twice the $x$-dimension. The kinetic energies in the $x$ - and the $y$-directions were then greatest for higher modes than unit wave numbers, indicating that this choice of computational box is acceptable. As another example for $\lambda=0$, we performed numerical simulations for a computational that was four times larger in the $x$ - and the $y$-directions than that used for the marginal state. From the calculations up to $R a=30$ $\left(R a=1.5 R a_{\mathrm{cr}}\right)$, it turned out that the kinetic and magnetic energies in the $x$ - and $y$-directions were still greater for the 3rd and 4th wave numbers, which means that the computational box was appropriate to the present study. A larger computational box might be even better, but would require more grid points and a much greater total integration time for the numerical simulations. The selection of the size of the computational box is a compromise between the need for physical realism on the one hand and the need to husband available computer resources on the other. 


\section{Trajectories of $\overline{\theta \mathbf{v}}$}
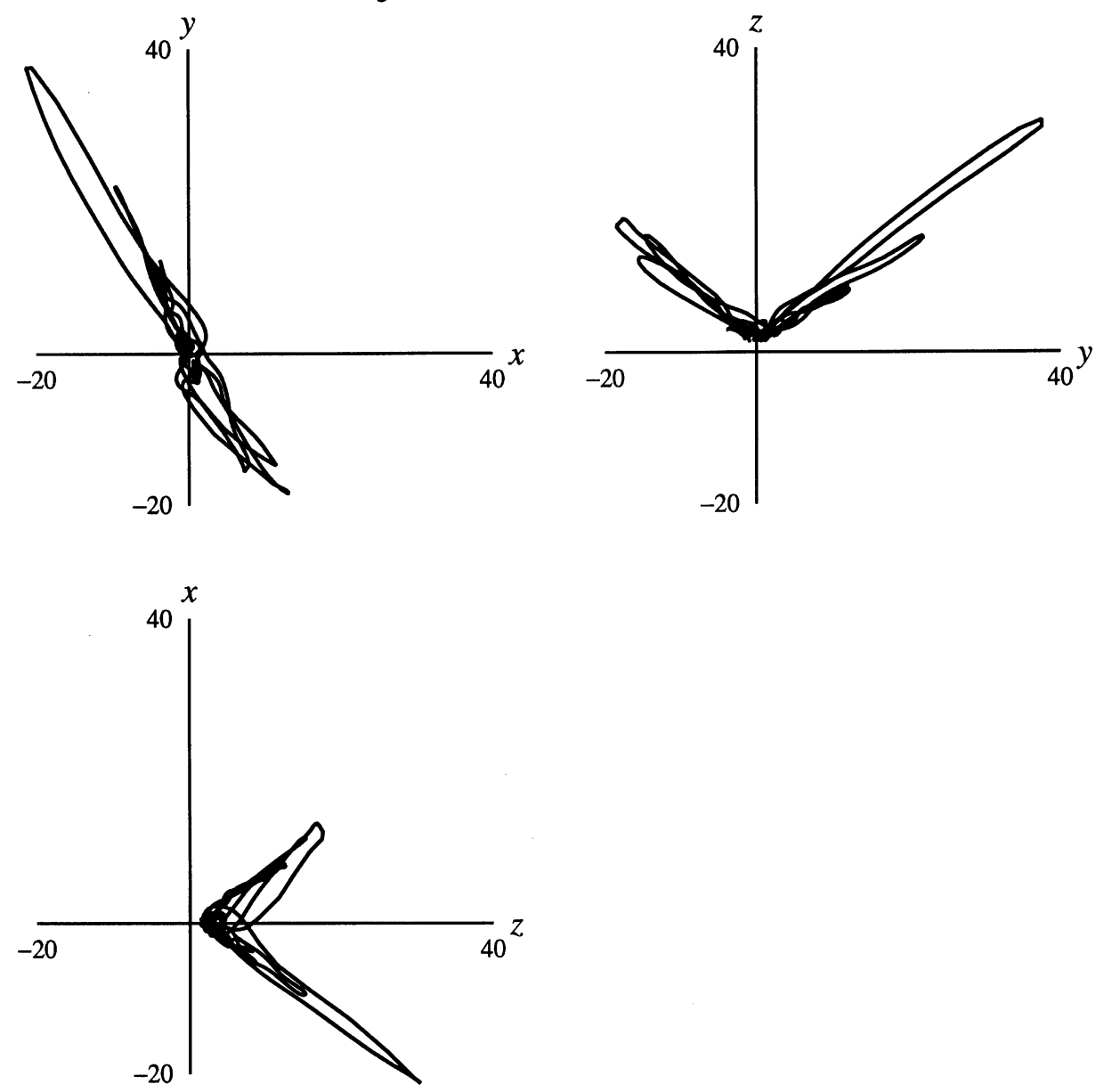

Fig. 7. Trajectories of the turbulent flux, $I_{x}, I_{y}$, and $I_{z}$ projected onto the $x y-, y z$ - and $z x$-planes for $\lambda=0$ and $R a=102$.

We performed numerical simulation for three choices of colatitude for the computational box: $\lambda=0, \lambda=\pi / 6$, and $\lambda=\pi / 3$. Table 2 summarizes the resulting turbulent flux, I. For $\lambda=0$ and $R a=40 \approx 2 R a_{\text {cr }}$, we took $\left(\ell_{x}, \ell_{y}, \ell_{z}\right)=(0.7660,3.766,1)$ which is tantamount to doubling in its $x$ - and $y$-dimensions the cell for which the growth rate is maximal when $R a=40$ and $\varepsilon_{v}=10^{-5}$. (For computational simplicity, we took $\varepsilon_{v}=10^{-3}$.)

Judging from the values of the standard deviations, it seems very likely that, when $\lambda=0$, both $\left\langle I_{x}\right\rangle$ and $\left\langle I_{y}\right\rangle$ vanish. This result is inconsistent with a conclusion of Braginsky and Meytlis (1990), who argued that $\left\langle I_{y}\right\rangle \sim\left\langle I_{z}\right\rangle$ and evidently $\left\langle I_{z}\right\rangle$ is nonzero. Although our result was derived from not as highly a turbulent state as they envisaged, we believe that our result is likely to be generally correct. When $\lambda=0$, a fluid parcel lighter (or hotter) than its surroundings should move antiparallel to gravity, and a parcel that is heavier (or colder) should move in the $-z$-direction. The Coriolis force does not deflect this motion, and the Lorentz force does change its direction. The existence of irregularities in $\mathbf{v}$ associated with the turbulence will of course mean that, at any particular time and point in space, $\theta v_{x}$ and $\theta v_{y}$ are nonzero, and that therefore the time and space average of $\theta v_{x}$ and $\theta v_{y}$ over any simulation will not be precisely zero. Nevertheless, as the duration of the simulation is continued, we may expect these averages will tend to zero. It seems to us that the ensemble mean turbulent flux, $\langle\mathbf{I}\rangle \equiv\langle\overline{\theta \mathbf{v}}\rangle$, is therefore in the $z$-direction when $\lambda=0$.

For $\lambda=\pi / 6$ and $R a=40 \approx 3.35 R a_{\mathrm{cr}}$, we took $\left(\ell_{x}, \ell_{y}, \ell_{z}\right)=(0.9624,2.925,1)$. As an example, we show in Fig. 5 the time evolution of $I_{x}, I_{y}$ and $I_{z}$. It is clear that $I_{x}$ and $I_{y}$ do not vanish. This may be explained in the following way. A fluid parcel lighter than its surroundings experiences a buoyancy force in the - g-direction, i.e., in the direction $\frac{1}{2}\left[\mathbf{1}_{x}+\sqrt{ } 3 \mathbf{1}_{z}\right]$. As it moves in response to this force, the Coriolis force deflects its upward motion into the $-y$-direction. For a heavier parcel the deflection is reversed, but so also is $\theta$. We therefore have $I_{x}>0$ and $I_{y}<0$. Also $\left|I_{y}\right|$ exceeds $\left|I_{x}\right|$. This is a consequence of the Lorentz force which hampers the motion of parcels in the $x$-direction, across the lines of force of the applied field, but not in the $y$-direction, which is parallel to the applied field.

For $\lambda=\pi / 3$ and $R a=40 \approx 4.43 R a_{\mathrm{cr}}$, we took $\left(\ell_{x}, \ell_{y}, \ell_{z}\right)=(2.302,2.819,1)$. In the same way as for $\lambda=\pi / 6$, a buoyant fluid parcel is driven by gravity, which is now in the $\frac{1}{2}\left[\sqrt{ } 3 \mathbf{1}_{x}+\mathbf{1}_{z}\right]$ direction. Coriolis and Lorentz forces act on its $x$-components of motion. As a result, $I_{x}>0$, $I_{y}<0$, and $\left|I_{y}\right|>\left|I_{x}\right|$. 


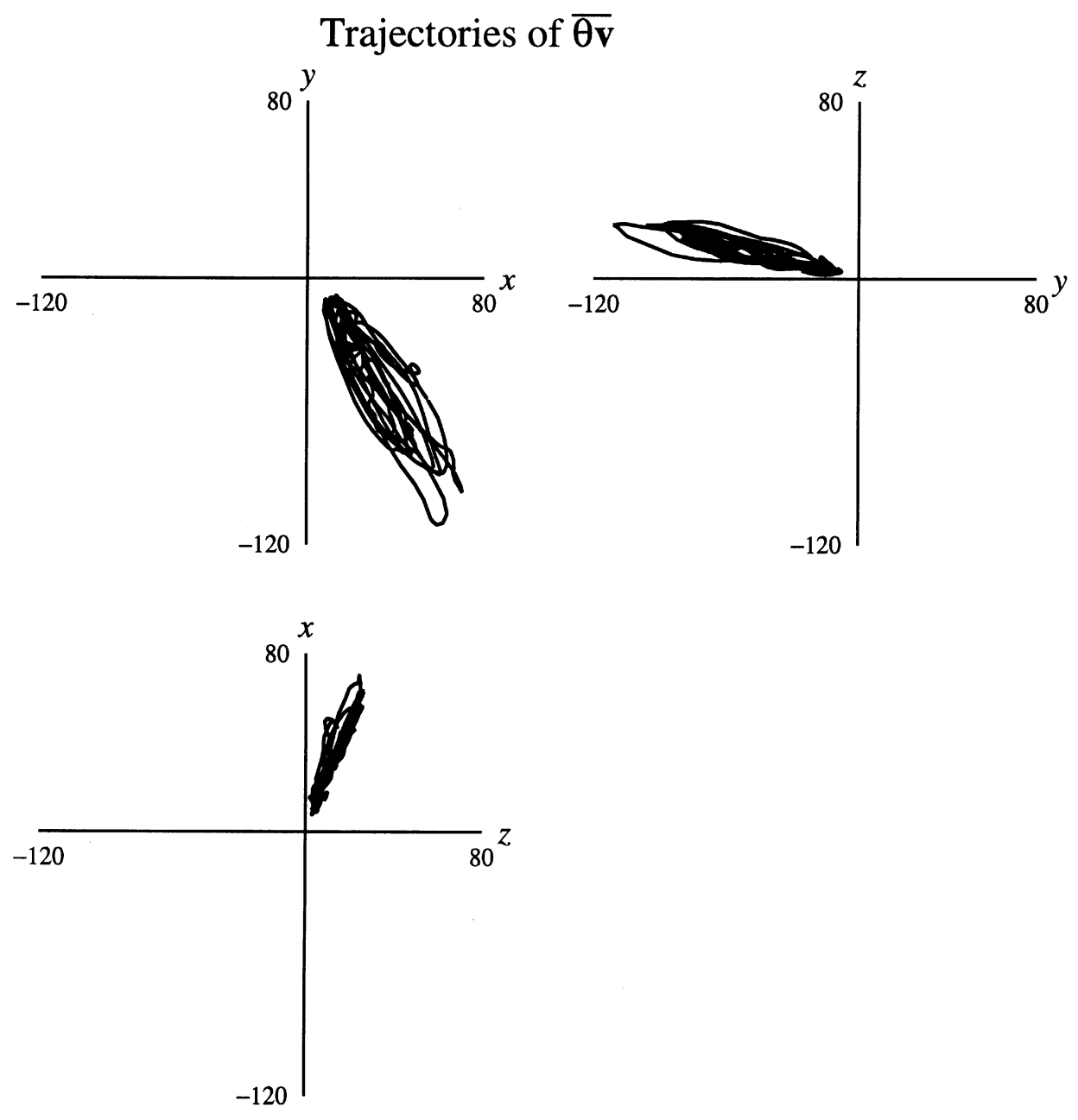

Fig. 8. Trajectories of the turbulent flux, $I_{x}, I_{y}$, and $I_{z}$ projected onto the $x y$-, $y z$ - and $z x$-planes for $\lambda=\pi / 3$ and $R a=120$.

We re-interpreted our time series for $I_{x}, I_{y}$ and $I_{z}$ in terms of the magnitude $I$ of $\mathbf{I}$ and its direction as defined by the angles $\Psi$ and $\Phi$, where

$$
\begin{aligned}
& I=\sqrt{ }\left(I_{x}^{2}+I_{y}^{2}+I_{z}^{2}\right), \\
& \Psi=\tan ^{-1}\left[\sqrt{ }\left(I_{x}^{2}+I_{y}^{2}\right) / I_{z}\right], \\
& \Phi=\tan ^{-1}\left(I_{y} / I_{x}\right) .
\end{aligned}
$$

The results are summarized in Table 3. It is interesting that $\langle\Psi\rangle>\lambda$ in all cases, and that the standard deviations for $\Psi$ and $\Phi$ are very small for $\lambda=\pi / 6$ and $\lambda=\pi / 3$. This implies that the turbulent flux, I, has a preferred direction, determined by the directions of the rotation axis, the imposed strong magnetic field and the gravitational force. The local values of the nondimensional parameters determine the magnitude $I$ of $\mathbf{I}$. We show in Fig. 6 an example of a trajectory of $I_{x}, I_{y}$ and $I_{z}$ for $\lambda=\pi / 6$. It is clearly seen how the turbulent flux I fluctuates about its preferred direction.

We have also examined turbulent transport for larger Rayleigh numbers for which the motions are more highly turbulent. For $\lambda=0, R a=102 \approx 5 R a_{\text {cr }}$ was used together with $\left(\ell_{x}, \ell_{y}, \ell_{z}\right)=(0.7520,1.729,1)$. The other nondimensional parameters were not changed. The components of the turbulent flux $\langle\mathbf{I}\rangle$ are shown in Table 2, and $\langle I\rangle,\langle\Psi\rangle$ and $\langle\Phi\rangle$ in Table 3. The standard deviation for $\Phi$, which is about $90^{\circ}$, arises because the turbulent transport is in opposite directions as seen in the $x y$-plane, as shown in Fig. 7 which, as in Fig. 6 , displays trajectories of $I_{x}, I_{y}$ and $I_{z}$. As mentioned earlier, a fluid parcel lighter than its surroundings should move predominantly in the $+z$-direction although fluctuations give it motion also in the $\pm x$ - and the $\pm y$-directions. Such flows perpendicular to the $z$-direction are influenced by the Coriolis force, and lead to turbulent transport is opposite directions.

For $\lambda=\pi / 3$, we took $R a=120 \approx 13.3 R a_{\text {cr }}$ and $\left(\ell_{x}, \ell_{y}, \ell_{z}\right)=(2.302,2.819,1)$. Values of $\left\langle I_{x}\right\rangle,\left\langle I_{y}\right\rangle,\left\langle I_{z}\right\rangle$, $\langle I\rangle,\langle\Psi\rangle$ and $\langle\Phi\rangle$ are given in Tables 2 and 3. It is clear that values of turbulent transport are much larger than those for $R a=40$. Even for this much more highly turbulent state, the turbulent flux again has a preferred direction, as shown in Fig. 8.

These values of the turbulent flux are much larger than the molecular flux for our value $\left(10^{-1}\right)$ of $P q$. This demonstrates that the effect of the small-scale turbulence on the diffusion processes of global fields is significant. Also the turbulent flux has a preferred direction. We should use this fact when we parameterize the anisotropic local turbulence in the Earth's core in simulating the geodynamo. 


\section{Concluding Remarks}

We have examined the anisotropy of local turbulence in the Earth's core by performing direct numerical simulations of thermally-driven magneto-turbulence in a rotating system. Our main focus has been the turbulent flux $\mathbf{I}=\overline{\theta \mathbf{v}}$, the average being taken over the small scale motions. We have found that, in the parameter ranges we have studied, this turbulent flux greatly exceeds the molecular flux and is therefore more effective in diffusing the global temperature field, $\bar{\Theta}$. We have demonstrated that this flux has a preferred direction that depends on colatitude in the core. The mean part of (3),

$$
\frac{\partial \bar{\Theta}}{\partial t}+\overline{\mathbf{V}} \cdot \nabla \bar{\Theta}+\nabla \cdot \mathbf{I}=\kappa \nabla^{2} \bar{\Theta},
$$

determines the evolution of $\bar{\Theta}$. The effect of turbulent transport $\nabla \cdot \mathbf{I}$ on the mean field is, in local turbulence theory, described by an anisotropic diffusivity tensor, $\boldsymbol{\kappa}^{t}$, as

$$
\mathbf{I}=-\boldsymbol{\kappa}^{t} \cdot \nabla \bar{\Theta}
$$

or

$$
I_{i}=-\kappa_{i j}^{t} \frac{\partial \bar{\Theta}}{\partial x_{j}}
$$

with the usual summation convention.

In this paper, we have assumed that $\nabla \bar{\Theta}$ is parallel to the gravitational vector, $\mathbf{g}$, so that we have focussed on only three, but three crucial, components, $\boldsymbol{\kappa}^{t} \cdot \mathbf{1}_{g}$, of the diffusion tensor $\boldsymbol{\kappa}^{t}$. To determine other components, it would be necessary to assume that $\nabla \bar{\Theta}$ and $\mathbf{g}$ are not parallel in our computational box. The character of the convection problem is then changed from one of convective stability to one of free convection. This raises fresh questions which were not addressed here.

For $\lambda=0, \mathbf{I}$ is parallel to $\mathbf{g}$, so that $\kappa_{x z}^{t} \sim 0$ and $\kappa_{y z}^{t} \sim 0$. We have evaluated $\kappa_{z z}^{t}=\left\langle\overline{\theta v_{z}}\right\rangle \neq 0$, and have found that it depends strongly on the local Rayleigh number. For $\lambda=\pi / 6$ and $\lambda=\pi / 3$, we have shown that $\mathbf{I}$ is not parallel to $\mathbf{g}$, and in special cases we have determined its direction relative to $\mathbf{g}$ in terms of angles $\Psi$ and $\Phi$. Since $\mathbf{g}$ is antiparallel to $\mathbf{1}_{r}$, these angles provide information about the off-diagonal elements $\kappa_{\theta r}^{t}$ and $\kappa_{\phi r}^{t}$ of $\boldsymbol{\kappa}^{t}$, relative to the diagonal element $\kappa_{r r}^{t}$.

We found that, for the value $10^{-1}$ of $P q$ used in this study, I greatly exceeds the molecular flux, which in dimensionless variables is $-\kappa \nabla \bar{\Theta}=P q \mathbf{1}_{r}$ (since $|\nabla \bar{\Theta}|=1$ in dimensionless units). This means that the effect of local turbulence on the diffusion of global fields is significant. Most elements of the anisotropic diffusivity tensor $\boldsymbol{\kappa}^{t}$ exceed $\kappa$, which suggests that local turbulence is important for the geodynamo.

We have considered a small region in the bulk of the core, and therefore periodic boundary conditions have been imposed. If a boundary exists, however, it is known that the velocity-gradient is large and wall turbulence tends to develop there (e.g., Arakawa, 1994). Very fine grids are then required, but a law for wall turbulence facilitates numerical computation. For the Earth's core, the role of wall turbulence in the presence of rotation and magnetic field will eventually have to be clarified. These effects are beyond the scope of the present study and remain to be solved in the future. Meanwhile, a realistic parameterization of local turbulence is important in modeling global geodynamo processes.

Acknowledgments. We are grateful to K. Zhang and H. Shimizu for their useful comments. Takahiro Nakajima was supported by the National Science Foundation, on Grant EAR94-06002. Some of the simulations were run on the Cray T3E at San Diego Supercomputer Center and on the SP2/cluster at OAC, UCLA.

\section{References}

Arakawa, C., Computational Fluid Dynamics for Engineering, 229 pp., Tokyo Univ. Press, Tokyo, 1994 (in Japanese).

Braginsky, S. I., Magnetohydrodynamics of the Earth's core, Geomagn. Aeron. (Engl. Trans.), 4, 698-712, 1964.

Braginsky, S. I. and V. P. Meytlis, Local turbulence in the Earth's core, Geophys. Astrophys. Fluid Dynam., 55, 71-87, 1990.

Braginsky, S. I. and P. H. Roberts, Equations governing convection in Earth's core and the geodynamo, Geophys. Astrophys. Fluid Dynam., 79, 1-97, 1995.

Glatzmaier, G. A. and P. H. Roberts, A three-dimensional self-consistent computer simulation of a geomagnetic field reversal, Nature, 377, 203209, 1995a

Glatzmaier, G. A. and P. H. Roberts, A three-dimensional convective dynamo solution with rotating and finitely conducting inner core and mantle, Phys. Earth Planet. Inter., 91, 63-75, 1995 b.

Kuang, W. and J. Bloxham, An Earth-like numerical dynamo model, Nature, 389, 371-374, 1997.

St. Pierre, M. G., On the local nature of turbulence in Earth's outer core, Geophys. Astrophys. Fluid Dynam., 83, 293-306, 1996.

Zhang, K., Nonlinear magnetohydrodynamic convective flows in the Earth's fluid core, Phys. Earth Planet. Inter, 111, 93-104, 1999.

Zhang, K. and C. A. Jones, The effect of hyperviscosity on geodynamo models, Geophys. Res. Lett., 24, 2869-2872, 1997.

M. Matsushima (e-mail: mmatsush@geo.titech.ac.jp), T. Nakajima (e-mail: takahiro@tono.jnc.go.jp), and P. H. Roberts (e-mail: roberts@ math.ucla.edu) 